\title{
norden
}

Nordic Council of Ministers

Ved Stranden 18

DK-1061 Copenhagen K

www.norden.org

NORDISKE ARBEJDSPAPIRER

NORDIC WORKING PAPERS

\section{Lessons from Nordic Council of Ministers study "Improving Nordic policymaking by dispelling myths on sustainable consumption"}

Oksana Mont, Lund University, Sweden

Eva Heiskanen, National Consumer Research Centre, Finland

Kate Power, Copenhagen Resource Institute, Denmark

Helka Kuusi, National Consumer Research Centre, Finland

NA2013:915

http://dx.doi.org/10.6027/NA2013-915

This working paper has been published with financial support from the Nordic Council of Ministers. However, the contents of this working paper do not necessarily reflect the views, policies or recommendations of the Nordic Council of Ministers. 



\section{NORDIC POLICY BRIEF}

Lessons from Nordic Council of Ministers study "Improving Nordic policymaking by dispelling myths on sustainable consumption".

Policy Brief, June 2013

\section{INTRODUCTION}

\section{Reason for this research}

Research goal

Research approach
Despite 20 years of policymaking on sustainable consumption (UNCED 1992), levels of material consumption and environmental impacts continue to increase in Nordic countries and the rest of Europe. As Nordic countries have an ambition to be sustainability leaders, enabling and facilitating sustainable consumption and lifestyles with efficient policies is an important part of the societal efforts to reduce resource use and environmental impacts. Clearly, however, current consumption policies are not as effective as they might be.

A number of reasons for this have been identified ranging from the principle of consumer sovereignty, to lack of policy instruments and missing political courage to engage with consumption issues. In addition, although a large share of environmental impacts depend on consumption patterns, research demonstrates that evidence from behavioural and social science is not routinely incorporated in policy design.

Consequently, some persistent misconceptions - myths - about consumer behaviour have perpetuated in the mainstream discourse on sustainable consumption especially in policy circles. Holding on to these myths encourages policy makers to place the main focus on technological innovation aiming at production and product efficiency, leaving social innovation, alternative value-creation models and sufficient consumption without much-needed support. These misconceptions prevent policy makers from seeing the complexity of consumption in real life, the limitations of the half-measures advocated by many sustainable consumption policies and therefore from effectively addressing the sustainability challenges associated with consumption.

The goal of this study is to dispel myths that thwart sustainability by bringing forward existing evidence on consumer behaviour, in order to aid the development of efficient sustainable consumption policies in Nordic countries.

To dispel the myths about consumer behaviour, the study builds on a substantial existing body of international research on consumer behaviour from psychology, sociology, and behavioural economics to policy and anthropology. This knowledge, however, has not reached the majority of policy makers working with sustainable consumption issues. The study uses a knowledge brokerage approach that exchanges and transfers the vast academic and practical multi-disciplinary knowledge between science, policy makers and practitioners in an accessible and easy-to-use format to enable and facilitate the application of existing knowledge in real life policy. 
Key messages for policy-makers
It is unrealistic to expect a sustainable society to materialise from current political strategies on sustainable consumption. The changes needed are significant, and the research explored in this study shows that policy makers have a plethora of opportunities to create positive change using strategies and tools synergistically.

Our society is consumptogenic: the structures of society promote consumption patterns that Nordic people think of as normal, but which are unsustainable. On the other hand, citizens who attempt to make significant lifestyle changes for sustainability face insurmountable socio-cultural barriers to sustainable practices. This highlights the need for governments to lead the shift to cultures of sustainability.

Governments need to lead the shift to sustainability by creating the societal structures that make sustainable living the default option. Innovation in technology and infrastructure, regulation, pricing, marketing and new social norms can be used in combination to create sustainable choice architecture.

Regulations are often the most effective policy tools for changing consumption patterns. Although regulations may be more challenging to implement, evidence is available showing practical techniques for successfully implementing stronger policy interventions. Regulations are often more effective when used in combination with other policy instruments, e.g. economic and information tools in policy packages.

Building positive social norms is essential for embedding sustainable practices in everyday life and for increasing public acceptability for stronger consumption policies. Even coercive, proscriptive policies that require significant lifestyle changes (e.g. switching from private car use to public transport) can gain higher public acceptance by using appropriate framing techniques, reinforcing pro-societal and pro-environmental social norms, and by providing safe, comfortable and cheap sustainable alternatives to unsustainable behaviours.

A policy focus is needed on facilitating change away from high-impact consumption areas (e.g. flying, consumption of meat and dairy products and car driving) to lower-impact consumption areas (e.g. vegetarian diets, public mobility, local leisure and cultural activities, and personal development).

Understanding and supporting the drive of humans to become happier and healthier, there is a need to discuss a much greater diversity of paths to wellbeing than is currently offered. It may be useful to communicate a wider vision of well-being, which includes pro-societal values such as resilient communities, equitable, fair and sustainable resource use, health, education and personal development, peace and stability, environmental and social justice and other macro-issues that indirectly influence individuals and families. To support and encourage sustainable ways of living new metrics of societal prosperity needs to be developed. 


\section{KEY LESSONS ON MYTHS}

Myth 1: Green consumption is the solution

Green consumption is one, but not the only strategy for reducing resource use and environmental impacts stemming from consumption

Myth 2: Consumers should lead the shift to sustainability

\section{There is a limit to what individual behavioural change can achieve. Systemic changes in the prevailing economic institutions and business models, regulations and infrastructures are required. Governments need to lead the transition to sustainability; individuals, business and civil society have other vital roles to play}

Truth: Green consumption is an important - but limited - strategy that aims to improve the efficiency of production processes and to design and sell environmentally and socially sound products.

Challenges: Increasing efficiency is essential and must continue; however, it is not sufficient: even 'green' products have impacts. Also increasing consumption levels and unsustainable consumption patterns outpace efficiency improvements achieved in production and product design stages. Thus, green consumption helps us to slow down the environmental problems, but cannot on its own prevent or avoid them.

The myth promotes the falsely optimistic view that technological solutions will be sufficient to achieve sustainability. It detracts from the need to tackle challenging issues, e.g. the prevailing culture of consumerism and materialism that is closely linked to resource use and environmental impacts.

Implications: Sustainable consumption and sustainable lifestyles strategies need to accompany efficiency strategies and could include such sufficiency strategies as e.g. shifting from products to consumption of services and sharing products instead of owning them, utilising the idling capacity of goods, and promoting a culture of creativity in upcycling and product repair.

Truth: Consumers have some responsibility for the consequences of their consumption and lifestyles, not least because households cause $40 \%$ of overall environmental impacts in the society. However, governments drive mass shifts in culture and consumption and production patterns.

Challenges: There is a false perception that consumers drive markets and businesses are just responding to demands. Both businesses and policy makers are choice architects, delimiting options for consumer actions. Dominant societal values, practices and social norms are shaped by policy e.g. through regulation, infrastructure, pricing mechanisms and the education system.

Implications: This study shows that several stakeholders, including businesses and consumers, call upon governments and policy makers to show leadership. Governments are the most significant agents for driving widespread changes in our culture of consumption; citizens, businesses and civil society have other vital roles to play.

Sustainability needs to be addressed as a fundamental necessity and not as a retail option. Currently people who try to live sustainably often find it hard to live in opposition to mainstream norms and practices. Some behaviours are "locked-in" by infrastructural constraints, pricing and social conventions. Policymaking has a key role to play in normalising sustainable lifestyles. It is necessary for governments to take the lead role in enabling sustainable lifestyles. 
Myth 3: If everyone does a little we will achieve a lot

\section{Everybody should contribute to sustainability, but big changes are needed to shift societies to sustainability}

\section{Myth 4: Small and easy environmental actions will} spill-over to bigger changes

The spill-over effects are only likely to happen between similar actions or contexts
Truth: Small changes are an important starting point. The "if everyone" slogan underlines that everyone's contribution is worthwhile and significant, and that others are also taking responsibility for their behaviour and making changes. Small changes are important, as people need to start somewhere, feel part of the group and feel they are doing something good. However, reaching a tipping point and establishing a new social norm is a significant challenge.

Challenges: The biggest misconception is that small individual changes will lead to significant results, since the sum of small individual changes usually only results in small aggregate improvements. Another issue is the framing of the slogan: people know that not everybody is "doing their bit" and might get discouraged from taking action. They often have difficulty seeing how small actions in the household may help solve global environmental problems and this leads to scepticism. "Free riding" might also be encouraged when people believe that their inaction will not have a major impact on the aggregate level. In addition to these problems, two types of rebound effects occur. A behavioural rebound effect takes place when people feel their small token actions (e.g. recycling paper) justify inaction in other areas, e.g. driving a car instead of taking public transport. A financial rebound effect occurs when people save money e.g. from cycling instead of driving, and spend the money on high impact activities e.g. long-distance holidays.

Implications: Small changes and mass movements make a difference, but it is essential to communicate that big changes are also needed. Positive encouragement and providing a realistic picture of the scale of change needed in society are also vital. Communication from policy makers and civil society should emphasise both the relative importance of the small changes that individuals can make in their life and the necessary largescale changes and the ways, in which citizens can participate in these.

Truth: Marketing theory encourages us to 'start where people are', get them moving in the right direction with an easy action, which should then theoretically make it easier to move up the next level of behaviour change. Research demonstrates that positive spill-over mostly occurs between similar actions, e.g. recycling at work may encourage recycling at home or buying eco-labelled products may spill-over to buying Fair Trade products.

Challenges: Positive spill-over is unlikely to lead to bigger changes. For example, small actions, such as recycling, do not usually spill over to the larger lifestyle changes that are needed, such as giving up use of a private car.

Implications: The spill-over effect may be more successful when people take a small action and then identify themselves as a person who cares about the environment. This can be encouraged by framing actions as "positive for the environment and society" rather than only as "saving money", and by encouraging change through empowering community initiatives.

While it is important to encourage people to take easy environmental actions to reduce their impacts, it is vital to realise that spill-over into significant lifestyle changes can hardly be expected. The significant lifestyle changes need to be incentivised, supported and facilitated by policies for sustainable infrastructure, pricing mechanism and sustainable marketing. 


\author{
Myth 5: More information \\ leads to sustainable \\ behaviour
}

\section{Information alone does not usually change behaviour, but it is a vital part of policy package}

Truth: Raising awareness and providing information is a necessary part of moving towards sustainable society. While information provision is a useful part of policy packages, on its own it rarely leads to behaviour change.

Challenges: People are not always "rational utility maximisers", who have access to information and the ability and time to weigh all pros and cons of their decisions and actions. In addition to information and awareness, consumer behaviour is steered by a range of factors, ranging from infrastructure, institutions, marketing and pricing to emotions, habits and norms. Changes in behaviour are unlikely if the different actors send contradictory messages, e.g. people need to live sustainable lives, but also need to "buy us out of the financial crisis". It is also challenging to facilitate sustainable behaviour through public information campaigns, which compete with commercial marketing and advertising in media, public spaces and on the Internet.

Implications: Sustainable behaviour needs to be promoted by congruent messages coming not only via information provision, but also through other strategies, e.g. infrastructure, marketing, pricing and societal institutions. There is a need to find ways to inform people about environmental problems so that it stimulates action rather than discourages them.

Information may have an effect on behaviour, but usually only when strong instruments are used at the same time, especially regulation and pricing. In addition, due to the attitude-behaviour gap, better results are sometimes reached when people are given the possibility to try the behaviour in addition to providing them with information. Change in attitudes follows once the behaviour is established.

Truth: Appealing to self-interest, e.g. promoting environmental actions as a way to save money, sometimes works well in the short term and for specific behaviours that are easy to link to increasing personal utility. However, appealing to self-interest defined in materialistic terms might inadvertently worsen the situation in the long term.

Challenges: Selling sustainability via self-interest may backfire when changes are needed that offer no immediate personal gain. Even the interests of future generations might be difficult to safeguard in a society that propagates immediate wins and prizes ruthless competition at the cost of the environment and social equity.

Implications: Policy makers should avoid sending mixed messages to citizens and not emphasise immediate personal gains when societal values are at stake. People are likely to behave in a more civic-minded way when pro-social values are emphasised, which could increase not only the acceptance of sustainable consumption policies, but also their effectiveness.

One possible solution is to expand the concept of self-interest to include spending time with family and friends, undertaking health-enhancing activities, engaging with community etc. Even social status can be linked to being a conscious consumer or living in voluntary simplicity, and not necessarily by leading stressful high-pay / high-impact career-oriented lives. To ensure a fair and equal sustainable society, we need to balance "What's in it for me?" with "What's in it for us?" 
Myth 7: Sustainability means "living in caves"

\section{Examples of sustainable living are emerging. We need a planned transition to sustainable lifestyles in order to avoid lower standards of living in the future}

Truth: Research demonstrates that an equitable share of greenhouse gas emissions would be around 2 tonnes $\mathrm{CO}_{2}$ per person per year, which implies much lower levels of material consumption for Nordic countries, which currently have 6-9 tonnes of $\mathrm{CO}_{2}$ per capita.

Challenges: It is utopian to think we can continue with business as usual, but the alternative - living sustainably - has been portrayed as living in hardship, leading a boring and inconvenient life, being pious, making sacrifices and having lower standards of well-being. This perspective makes it hard to promote sustainable lifestyles and create acceptance and public engagement. It also undermines the opportunity to promote productsharing schemes or closed loop systems that could reduce overall resource use and environmental impacts associated with consumption without affecting well-being.

Implications: Examples of sustainable living are emerging. We need a planned transition to sustainable lifestyles in order to avoid lower standards of living in the future.

There is a need to better understand the benefits of low-impact lifestyles for the well-being of individuals and the society, as well as for the environment, and to advocate them based on criteria of good health, high quality of life and sufficient material consumption, rather than on merely material abundance. There is also a need to better communicate the consequences of inaction, without being dramatic but rather pragmatic, demonstrating that slow action is already leading to environmental problems (luckily not yet very visibly in Nordic countries) and reducing standards of living in many European countries.

The role of innovative value-creation business models, e.g. servicizing, in enabling sustainable living needs to be better understood. Both governmental and business support is needed to the growing community of individuals, municipalities and cities that enable more sustainable ways of living through social innovation, e.g. low-carbon communities and collaborative consumption.

Truth: There is a strong belief in our societies that having more money and owning more material goods brings us more happiness and well-being. Research demonstrates that up to a certain point, GDP increase is closely linked to growth in subjective well-being. Above a certain threshold, increases in GDP lead to reduced marginal levels of improvement in wellbeing.

Challenge: Individual levels of well-being are influenced more by wealth relative to others in society than to absolute levels of wealth. Some studies also demonstrate that it is possible to achieve higher levels of well-being with lower levels of material consumption once a certain level of material standard has been reached.

People pursue higher incomes and higher levels of material consumption in an attempt to increase personal happiness, even when this sometimes entails sacrifices to health, leisure time and family and community life. On the other hand, people who voluntarily choose to work and spend less often report increased levels of well-being and life satisfaction.

Implications: Politicians fear alienating citizens with policies that tackle 
consumption patterns and levels; perhaps framing policies and actions as promoting sustainable lifestyles could de-dramatise the focus on levels of material consumption and move the societal debate into a more proactive and productive course on how people's quality of life can be improved. Understanding and supporting the drive of humans to become happier, there is a need to discuss a much greater diversity of paths to happiness than is currently offered, e.g. reaping the benefits of technological progress not only in monetary terms, but also in terms of meaningful leisure activities and personal development.

To support and encourage sustainable ways of living new metrics of societal prosperity needs to be developed, e.g. alternative indicators to GDP developed by the WAVES World Bank programme and Beyond Growth movement.

Myth 9: Private ownership of all kinds of products is desirable - sharing is not

A sharing economy and
collaborative consumption
of all kinds of products are
making a revival. Policy
makers can help by
reducing barriers to a
sharing and collaborative
economy and by supporting
research needed on its
effects

A sharing economy and collaborative consumption of all kinds of products are makers can help by reducing barriers to a sharing and collaborative research needed on its effects
Truth: Our society is built on the institution of ownership. Sharing is seldom desired when the costs of sharing, either in terms of money or in terms of time spent on managing the sharing, are higher than costs of ownership.

Challenges: Many people are concerned about the growing number of products that take up space in their homes and that take time for their maintenance. The rate of product replacement is increasing, and there are rising consumption levels of poor quality, cheap throwaway products.

Although product ownership is increasing, collaborative consumption of all kinds of products is also making a revival, for example through swapping events, online trading of second-hand goods, community libraries for equipment, and city rental schemes for cars and bicycles. The Nordic countries have several traditional systems for sharing products, such as public libraries and common laundry facilities, which can be enhanced by new innovative forms of collaborative consumption.

Implications: Many innovative new businesses demonstrate that there is potential to increase sharing and redistribution of existing resources and products. Reusing products helps avoid environmental impacts associated with production of new products and urban mining is seen as an important future source of materials. A sharing economy can be supported through formal and informal initiatives for lending, swapping, bartering and renting products and services, instead of individual product ownership. 
Myth 10: Consumption

policies are too

controversial to be accepted

by the public

\author{
Policy is never neutral, but it \\ shapes social norms and \\ values in society. Policy \\ makers need to create the \\ "politics of possibility" \\ towards sustainability by \\ using a plethora of existing \\ and new strategies and tools \\ synergistically
}

Truth: Sustainability policies focusing on consumption do not have as long a history as many other fields of environmental policy. Consumption is often perceived of as being part of the private sphere of life, in which policy makers should not intervene. Consumers are assumed to be sovereign, i.e. they can make choices freely in the market, and those choices reflect their preferences. Hence, policy makers are often concerned that consumption policies are too controversial to propose, even where there is a strong public interest in influencing consumption patterns.

Challenges: There is no policy that is not value-laden. Many existing policies already influence our individual lifestyles and consumption - we are just accustomed to them, so they are 'invisible' e.g. drink driving laws, wearing seat belts, etc. Regulation of consumption has been effective even when initially being considered too controversial e.g. smoking bans, congestion charges, the phase-out of inefficient light bulbs and others.

Implications: Despite a reluctance to be interventionist or paternalistic, policy is never neutral: it shapes social norms and values in society. For example, strict targets on greenhouse gas reductions can communicate the seriousness of the environmental issue to the population.

There are several strategies that are known to increase the acceptance of sustainable consumption policies, e.g. building positive social norms supporting stronger policy interventions, the possibility for citizens to experiment and gain experiences of the measure, the involvement of citizens in the decision, the achievement of visible positive effects and the adaptation of attitudes once the measure is normalised. Thus, the way a policy is communicated and implemented can greatly increase public acceptance, even for more challenging behaviours and proscriptive policies.

Nordic policy makers can gain inspiration from J.F. Kennedy's decision to put a man on the moon, which was, when Kennedy first announced it, ridiculed as 'impossible'. To achieve the impossible, he created a culture of possibility, which supported previously incomprehensible and unforeseeable technological breakthroughs. The effort of putting a man on the moon implied systemic and visionary shifts that required the support of multiple governmental departments and many sectors that tapped into deep cultural values to create the 'politics of possibility'. 


\section{KEY LESSONS ON KNOWLEDGE BROKERAGE}

One of the key barriers to efficient evidence-based sustainable consumption policymaking is the persistence of misconceptions, simplifications and generalisations about consumer behaviour: what we describe here as myths. One of the complexities is that all myths have some element of truth. Thus, the main issue is to find and present available evidence in an objective and balanced way. Another problem is that research on consumer behaviour is indeed complex and might be difficult to utilise in the policy realm, especially as the majority of decision-makers who devise policies for sustainable consumption have backgrounds in economics, engineering, law, or natural science, while those with background in behavioural science are underrepresented. As a result of this structural lock-in, evidence from behavioural sciences is insufficiently utilised.

This study ventured into the vast multi-disciplinary body of knowledge on consumer behaviour and collected and presented evidence about the aforementioned misconceptions. In addition, this study identified barriers associated with current knowledge brokerage and outlined the best means to make knowledge provision, sharing and exchange more accessible. These insights were gained from interviews with Nordic policy makers and should help reduce the barriers to efficient sustainable consumption policy design and implementation.

How research results are used in policymaking
Time is the main constraint for Nordic policy makers to use results of research. This situation suggests a need for synthetic information that is simplified and rendered useful for policymaking. The knowledge interest of researchers and policy makers is often quite different. While researchers aim to challenge the existing views and create new knowledge, policy makers are looking for knowledge that is easy to use and that offers clear solutions.

Policymaking is also not merely a process of applying research, but a complex political process. Therefore, research needs to be convincing not only for the civil servants, but also for politicians and their electorates. This was often mentioned as one of the key areas where research falls short of expectations. Several interviewees stressed that the lack of societal and political consensus was often a barrier to applying new knowledge.

Most of the interviewees noted that law and economics were the two research disciplines that are used in an established way in policymaking. Environmental policy also often makes use of natural and engineering sciences, which were also seen as important for developing sustainable consumption policies. The greatest problems in knowledge integration were found in the social sciences, which are often not cumulative or contextindependent in the same way as the natural sciences are. Consumer behaviour comprises various types of behaviours occurring in different types of contexts, so there is no grand theory or strict laws to draw on.

Many of our interviewees stressed the need for more multidisciplinary research; in particular on how consumer behaviour can be changed and on the role of public policy for consumption in society, e.g. on the issue of consumption and its links to happiness and well-being. 


\author{
Availability of sufficient \\ research for \\ policymaking on \\ sustainable consumption
}

The main knowledge gaps identified by Nordic decision makers can be summarised as follows:

- Inconclusiveness: research is inconclusive, contradictory and consumer behaviour is not fully understood.

- Complexity: information is not collected and presented in an easy to understand form, which leads to its invisibility and lack of use by policy makers.

- Lacking prompt access: Many interviewees felt that it is difficult to promptly access the necessary information at the time when it is needed.

- Lack of applied research and policy assessment: Several interviewees stressed that there is limited evidence available on policy effectiveness. This is partly due to a lack of policy monitoring and evaluation, but also due to the lack of established methods for evaluating the effectiveness of policy instruments and measures.

- Lack of country-specific research: This is more of a problem for some countries than for others. Clearly the context influences the effectiveness of policy measures and studies are needed that would test applicability of policy measures in different contexts.

- Lack of dedicated research: Since sustainable consumption policy is quite a new policy area, it is difficult to identify what research is relevant and how the existing research should be combined to best serve the needs of policymaking. The larger the addressed societal problems, the greater the need is for engaging with research from several disciplines, although research funding does not always support the multidisciplinary needs of sustainable consumption research.

Most of our interviewees felt that there is insufficient knowledge for evidence-based policymaking, especially studies on policy interventions, which are often inconclusive. They emphasised the need for more impact studies, so that policy could be based on scientific evidence.

The areas where evidence is most lacking are economic impacts and effective ways to bring about changes in consumer behaviour. Often a lack of time and money is the reason why policies are not tested, monitored or evaluated, and the results of the few available studies are not used. However, the evidence-base is stronger in some areas than others, e.g. there is quite some evidence on public recognition and trust of the Nordic Swan label, but not much on other instruments.

Moreover, some of our interviewees felt that the lacking evidence-base is not the greatest problem, as such. Rather, they stressed the role of politics in policymaking, as well as the counter-forces to sustainable consumption policy, such as lobbying groups.

The Nordic interviewees have identified two main categories of barriers: ideological/political and practical barriers. They stressed the importance of the role of politics in policymaking as different ministries, political parties and agencies have different priorities, e.g. impacts on job creation or tax revenues. The main barriers however stem from a combination of the political factors and the way in which policy is made in practice. The Nordic
Barriers to knowledge use and ideas for surmounting them 
policy makers also suggested that improving the knowledge base and especially knowledge utilisation practices in the political deliberation process can perhaps help to surmount the ideological and political problems. They also offered some ideas for overcoming the barriers.

Several arguments were put forth for more co-operation among ministries. This was seen as very important because different policy instruments are often the mandate of different sectors of government, and it is difficult in such a situation to design an effective policy mix. Certain aspects of consumption (such as housing) may be better covered by existing administrative structures than others. The lack of policy coordination and the existence of contradictory pressures make decision making difficult.

In terms of political leadership, it was suggested that researchers should communicate more directly to politicians and the public: civil servants cannot easily go ahead of the political process, but receive their mandate to develop policies from politicians. On the other hand, researchers might highlight the limitations of consumer sovereignty and the fact that citizens actually value political leadership. However, some interviewees noted that the results communicated by researchers to the public are often contradictory and hence do not serve to promote political leadership in sustainable consumption.

The webinars undertaken as part of this study were very positively viewed by participants and so could serve as a useful model for future dialogue between researchers, policy makers and other stakeholders. The participants appreciated the 'innovative way to exchange ideas', the opportunity to make contact with new and old colleagues, the possibility to update their knowledge and to receive the final results of the study, to which they have contributed through the webinars. This might increase the willingness to use the research results.

This study confirmed that much of the existing body of knowledge on consumer behaviour is complex, nuanced and context-dependent, suggesting the need for new institutions for knowledge brokerage.

- a hub for knowledge brokerage, e.g. hosted by the Nordic Council of Ministers, which would serve to identify relevant studies from trusted sources, assess the quality of scientific information and communicate up-to-date knowledge to national governments.

- a 'consensus panel' among researchers for synthesising and presenting the main findings from consumption research - something in line with what the International Panel on Climate Change has done.

- a think tank or forum to bring together the scientific community with consumer organisations. Such a forum could be built on the values of solidarity and social democracy pertinent to the so-called Nordic model, and would explore a global perspective, discussing the consequences of consumption not only for the Nordic consumers, but also for consumers and countries outside Nordic borders.

Practical suggestions for presenting research results included calls for simple explanations of complex issues in accessible formats, illustrations and diagrams, good summaries of 2-4 pages and clear conclusions.

Research results should be presented forcefully and briefly, as the business lobbies do at the European Commission. 


\section{RESEARCH PARAMETERS}

Objective of the research

\section{Research design}

Research results
The specific objective of the study is to improve knowledge of Nordic policy makers on:

- Key myths about consumer behaviour that hinder effective policymaking on sustainable consumption,

- Key lessons from multi-disciplinary research on consumer behaviour and consumption,

- Implications of the new knowledge gained for policy implications.

This is a 1-year knowledge brokerage study that consisted of four stages:

Stage 1: A desk-top study included literature meta-analysis of the existing body of knowledge on each myth according to the following structure: 1) its origins; 2) consequences for society, consumption patterns and levels, actors and policy actions; 3 ) arguments, evidence and data that dispel each myth; and 4) implications for existing and future policy strategies.

Stage 2: The empirical study included collection of data through semistructured interviews with 22 Nordic policy makers and experts about myths on consumer behaviour and their implications for policymaking to promote sustainable consumption. Two focus groups were conducted with 10 researchers in the sustainability field and with 8 citizens, members of the Swedish Association of Sustainability Psychology.

Stage 3: Analysis and synthesis brought together the academic research with the real-life experience of policy-makers in the field of sustainable consumption. It provided a comprehensive evaluation of the issues relevant for sustainable consumption policymaking.

Stage 4: Dissemination and finalisation of findings included soliciting feedback from the target group - primarily Nordic policy makers - through a webinar. A second webinar was also organised to test the accessibility of the knowledge and findings on a group of SCP researchers and practitioners, civil society experts and students from Europe and USA. In total 68 people participated in the webinars. The second webinar provided additional feedback about some of the more nuanced and controversial issues, and added strength and validity to the final findings of the study.

1. Final report in Swedish and English http://www.norden.org/en/nordiccouncil-of-ministers

2. Policy brief in English and Nordic languages 


\section{PROJECT IDENTITY}

\section{Coordinator}

\section{Consortium}

NCM contact

\section{Duration}

Funding Scheme

\section{Budget}

Website

Further reading
International Institute for Industrial Environmental Economics Lund University P. O. Box 196 Tegnersplatsen 4, SE- 22100 Lund Sweden

http://www.iiiee.lu.se/

Tel: +46462220250

Fax: +46462220230

Oksana Mont, Project Manager: oksana.mont@iiiee.lu.se

Oksana Mont, Lund University, Sweden Eva Heiskanen, National Consumer Research Centre, Finland Kate Power, Copenhagen Resource Institute, Denmark Helka Kuusi, National Consumer Research Centre, Finland

Camilla Sederholm +358400930886

E-post: camilla.sederholm@miljo.fi www.norden.org/hkp

15 May 2012 to 15 April 2013

Nordic Council of Ministers' working group on SCP

NCM contribution 330000 DKK

http://sustainabilitymyths.blogspot.se/

Final report of the study published online http://www.norden.org/en/nordic-council-of-ministers 